\title{
Commentary: Meaningful partnership with our intensive care medicine colleagues-The time is now
}

Judson B. Williams, MD, MHS, ${ }^{a}$ and Daniel L. Fox, MD ${ }^{\mathrm{b}}$

\footnotetext{
From the a Cardiovascular and Thoracic Surgery, WakeMed Health and Hospitals, and Duke University School of Medicine, Raleigh, NC; and ${ }^{\mathrm{b}}$ Department of Pulmonary and Critical Care Medicine, WakeMed Health and Hospitals, Raleigh, NC.

Disclosures: Authors have nothing to disclose with regard to commercial support.

Received for publication March 31, 2019; accepted for publication April 1, 2019; available ahead of print May 31, 2019.

Address for reprints: Judson B. Williams, MD, MHS, Cardiovascular and Thoracic Surgery, WakeMed Health and Hospitals, Duke University School of Medicine, 3000 New Bern Ave, Suite 1100, Raleigh, NC 27610 (E-mail: judson.williams@duke.edu).

J Thorac Cardiovasc Surg 2020;159:1378-9

$0022-5223 / \$ 36.00$

Copyright (c) 2019 by The American Association for Thoracic Surgery

https://doi.org/10.1016/j.jtcvs.2019.04.009
}

Over the last decade, "intensivists," dedicated physicians specializing in critical care medicine, are increasingly participating in the postoperative care of patients undergoing cardiac surgery. This trend has been postulated to improve adherence to evidence-based processes of care, shorten hospital and intensive care unit (ICU) stays, and reduce postoperative complications, especially pulmonary complications. However, the data to date supporting this practice of joint postoperative management have been sparse in the cardiac surgery literature. As such, we read with interest and appreciation the study by Huard and colleagues. ${ }^{1}$

Examined in the study were 16,454 patients over an 8-year period before and after implementation of 24-hour intensivist coverage. Pulmonary outcomes including costsaving and clinically meaningful markers of time on mechanical ventilation, rates of reintubation, and need for tracheostomy among matched patients were improved in the 24-hour intensivist coverage cohort and among high-risk patients (European System for Cardiac Operative Risk Evaluation $>5 \%$ ). Most important, both ICU and inhospital mortalities were significantly reduced. ${ }^{1}$

As a specialty, critical care remains in its infancy, yet it has grown quickly because of the increase in volume of critically ill patients and the growing clinical evidence for its beneficial role. Critical care medicine began to evolve into its current form in the 1940s and 1950s, arising out of necessity when appropriate airway and ventilator management were found to reduce mortality during polio epidemics. ${ }^{2}$ Intensivist staffing has been shown to reduce the length of ICU stay for a variety of medical and surgical disease processes, thus reducing the morbidity and mortality associated with prolonged ICU stays. ${ }^{3}$

Intensivists caring for the study patients hailed from a variety of subspecialties including anesthesiology $(n=7)$, respiratory medicine $(\mathrm{n}=5)$, cardiology $101(\mathrm{n}=2)$, nephrology $(\mathrm{n}=1)$, internal medicine $(\mathrm{n}=1)$, and cardiac

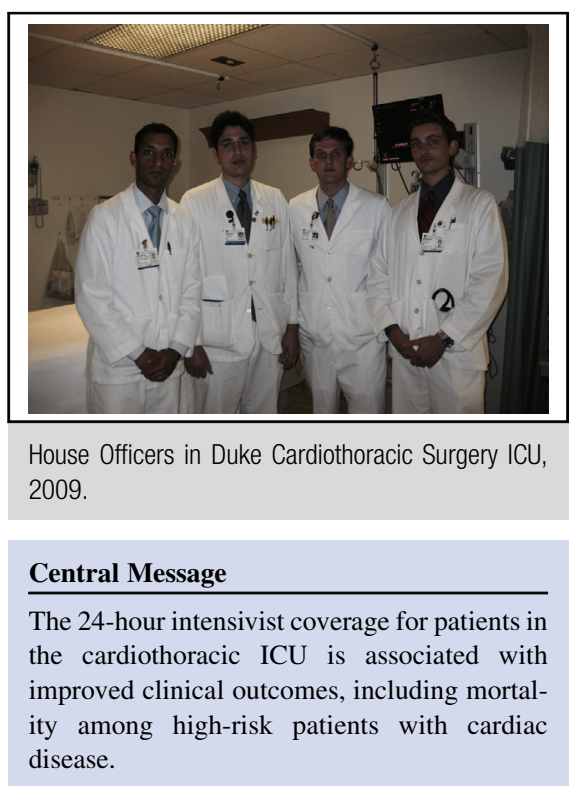

See Article page 1363. surgery $(\mathrm{n}=1)$, each with a board certification in critical care medicine. This diversity of background is a strength that the intensivist-staffing model provides, specialists with discrete and specific critical care training able to bring time and expertise to the intensively ill patient.

This model of shared, collaborative care for a complex cardiac surgery patient population is a relatively new idea. As recently as the 1990s, the American College of Surgeons guidelines for standards in cardiac surgery, developed by the Advisory Council for Cardiothoracic Surgery, stated that "only the cardiac surgeon was fully cognizant of all aspects of a given patient's condition and thus should be primarily responsible for the postoperative care of their patients." ${ }^{4}$ This included the multifaceted and dynamic care provided in the ICU. ${ }^{4}$ However, as patients presenting for cardiothoracic surgery have become older and increasingly comorbid, the merits of having attentive, continuous 24-hour care throughout the perioperative period have begun to be realized.

The nuanced medical management required and the direct attention to the complex and often tenuous respiratory mechanics of the perioperative cardiac patient offer benefit for an intensivist with expertise in cardiopulmonary physiology and respiratory mechanics. Huard and 
colleagues ${ }^{1}$ further this understanding and the benefits that 24-hour intensivist coverage could provide to the cardiac surgery patient population. Although concerns remain regarding the risks of discontinuity of care resulting from physicians guiding postoperative recovery without participation in the preoperative or intraoperative care of the patient, the trend toward collaborative, multidisciplinary care including critical care specialists is an undeniable compliment to the unique knowledge, expertise, and continuity provided by the surgeon.

With an eye to the future, a collaborative staffing model between cardiothoracic surgeons and a dedicated intensivist specialty team is increasingly evidence supported and steadily gaining wider adoption. The work by Huard and colleagues ${ }^{1}$ pushes forward the potential benefit of a multimodal, synergistic approach to continue to improve postoperative care for patients undergoing cardiac surgery. For better or worse, bygone are the days of house officers managing post cardiac surgery patients on nights and weekends because of the higher observed complications and mortality among this group.

\section{References}

1. Huard P, Kalavrouziotis D, Lipes J, Simon M, Tardif M-A, Blackburn S, et al. Does the full-time presence of an intensivist lead to better outcomes in the cardiac surgical intensive care unit? J Thorac Cardiovasc Surg. 2020;159:1363-75.e7.

2. Berthelsen PG, Cronqvist M. The first intensive care unit in the world: Copenhagen 1953. Acta Anaesthesiol Scand. 2003;47:1190-5.

3. Pronovost PJ, Angus DC, Dorman T, Robinson KA, Dremsizov TT, Young TL. Physician staffing patterns and clinical outcomes in critically ill patients: a systematic review. JAMA. 2002;170:2151-62.

4. Guidelines for standards in cardiac surgery. In: Bulletin of the American College of Surgeons. Chicago, IL: Advisory Council for Cardiothoracic Surgery; 1997: $82: 2$ 